\title{
IMPLANTABLE MEMS DRUG DELIVERY DEVICE FOR CANCER RADIATION REDUCTION
}

\author{
Heidi Gensler ${ }^{1}$, Roya Sheybani ${ }^{1}$, Po-Ying Li $^{1}$, Ronalee Lo ${ }^{1}$, Sutao Zhu ${ }^{2}$, Ken-Tye Yong ${ }^{2}$, Indrajit \\ Roy $^{2}$, Paras N. Prasad ${ }^{2}$, Rizwan Masood ${ }^{1}$, Uttam K. Sinha $^{1}$, Ellis Meng ${ }^{1}$ \\ ${ }^{1}$ University of Southern California, USA \\ ${ }^{2}$ University at Buffalo, The State University of New York, USA
}

\begin{abstract}
We present the first implantable MEMS drug delivery device that includes an electrochemical bellows pump, refillable drug reservoir, and dual regulation valve. Multiple drug pump configurations were fabricated, assembled, and tested. Delivery of agents for cancer radiation reduction was demonstrated. In vivo chronic delivery of radiation sensitizing agents in the form of small interfering (siRNA)-gold nanorod complexes (nanoplexes) directly to tumors induced in mice was achieved. Radiation therapy in conjunction with active drug pumping by electrolysis actuation resulted in significant reduction of colon cancer tumor (HT29) size $(\sim 50 \%)$ over diffusion-based delivery and intravenous injections. To our knowledge, this is the first MEMS drug delivery pump suitable for safe, efficacious, and local delivery of short half-life siRNA in vivo.
\end{abstract}

\section{INTRODUCTION}

Chronic delivery of therapeutics with spatiotemporal resolution ensures both adequate bioavailability and therapeutic efficacy but remains a medical challenge. Cancer treatment often involves radiation therapy which results in severe systemic side effects; reduction of the dose of ionizing radiation required for therapeutic efficacy is sought to limit damage to normal tissues. However, tumor cells may exhibit resistance to radiation-induced damage. The protein sphingosine kinase-1 (SPK-1) is implicated in the radioresistance of tumor cells [1]. By silencing the gene expressing SPK-1, it is possible to sensitize cells to radiotherapy and thus decrease ionizing radiation dosage. Gene silencing technology using siRNA has emerged as a promising therapeutic modality for a number of diseases including cancer, but the lack of a suitable delivery method has limited its use to date [2].

We have developed a MEMS drug delivery pump that allows direct delivery of siRNA-gold nanoplexes (HNB001) targeting the gene expressing SPK-1 to tumors (Figure 1). Electrostatic interactions between the gold nanorods and siRNA result in formation of nanoplex structures that facilitate cellular uptake of the siRNA. These combined events block the formation of SPK-1 leading to radiation-induced apoptosis in tumors for lower doses of ionizing radiation.

Several commercial drug delivery devices exist but are not suitable for our application, which requires periodic infusion of a drug bolus under low power operation in small animals (mice). The osmotically-driven Alzet ${ }^{\circledR}$ pumps are suitable for use in mice but provide only continuous infusion; the flow rate is specific to the particular model and cannot be altered following implantation. Delivery duration varies from 1 day to 6 weeks, and is therefore not adequate for extended chronic studies or therapies [www.alzet.com/]. The Med-E-Cell Infu-Disk ${ }^{\mathrm{TM}}$ is too large for practical implantation in mice and the flow rate is factory set. In addition, the disposable device requires a battery and can only be used once for continuous drug delivery [www.medecell.com/]. Other conventional drug delivery methods include tether infusion and vascular access ports, but these require frequent handling of laboratory animals and constant care to prevent infection and other complications. Many MEMS pumps have been reported but none are refillable and meet the low power requirements of our application.

\section{DESIGN}

Our drug delivery approach utilizes low power electrolysis actuation to deliver drug from an adjacent reservoir through an integrated cannula directly to the site of therapy (Figure 1). The operation principle and refill method are summarized in Figure 2. A bellows actuator format [3] was adopted to separate the drug from the electrolyzed fluid and prevent associated drug degradation, an undesirable effect reported in our previous work [4]. The electrochemically-actuated bellows imposes a pneumatic driving force on drug contained in the adjacent reservoir, forcing it out through the delivery cannula. Both wire and wireless actuator operation were previously demonstrated [5]. Integration of a check valve in the drug delivery cannula with bandpass flow regulation ensures on-demand and safe dosing [6].

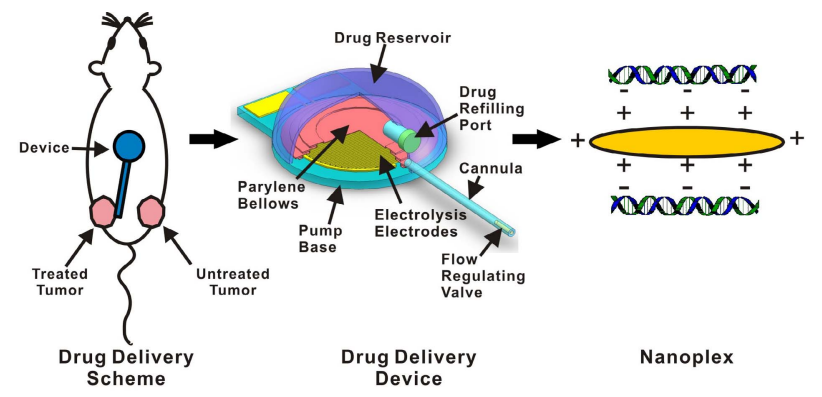

Figure 1: Left to right: Conceptual depictions of placement of implanted drug pump to deliver nanoplexes to tumors, the drug delivery system and components, and the nanoplex that delivers siRNA.

Electrolysis was chosen as the actuation method for its low power consumption $(\sim \mu \mathrm{W}$ to $\mathrm{mW})$, low heat generation, large driving force, and flow rate control through adjustments of the applied current. The electrolysis actuator consists of a pair of interdigitated electrodes, electrolyte (in this case, deionized (DI) water), 
and a flexible pressure transmitting membrane. A flexible bellows was chosen over corrugated membranes and flat membranes; higher deflections were achieved with bellows while imposing lower material stress under pressure loading $[3,5]$.

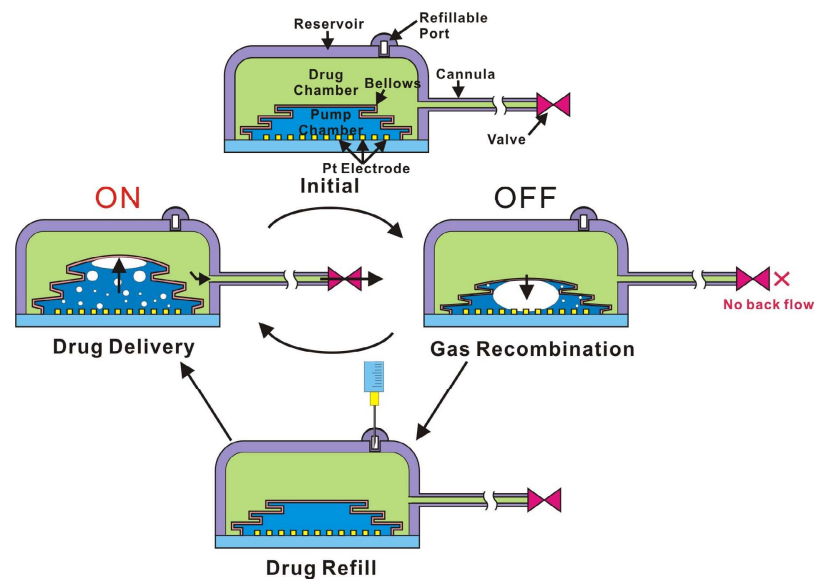

Figure 2: Illustration of drug delivery device operation.

\section{FABRICATION AND ASSEMBLY}

\section{Electrolysis Actuator Fabrication and Assembly}

Electrolysis electrodes were fabricated on thin film Parylene $\mathrm{C}$ membranes (5 $\mu \mathrm{m}$ thick) supported on a silicon substrate then released. Pt $(2000 \AA)$ and $\mathrm{Pt} / \mathrm{Ti}$ (2000 $\AA / 500 \AA)$ electrodes were patterned using a standard liftoff process described in [3, 5]. Following removal from the supporting $\mathrm{Si}$ substrate, electrodes were mounted on a flexible polyetheretherketone (PEEK) substrate (Figure 3). Parylene bellows were fabricated using a polyethylene glycol (PEG) molding process described previously [3,5]. Briefly, a silicone rubber master mold was used to cast PEG bellows forms. Parylene C $(10 \mu \mathrm{m})$ was deposited over the PEG form, and then the PEG was dissolved in hot DI water $\left(85^{\circ} \mathrm{C}\right)$. The Parylene bellows was filled with DI water and adhered to the electrode substrate with double-sided pressure sensitive adhesive film (Figure 3). The seal was further reinforced with epoxy.

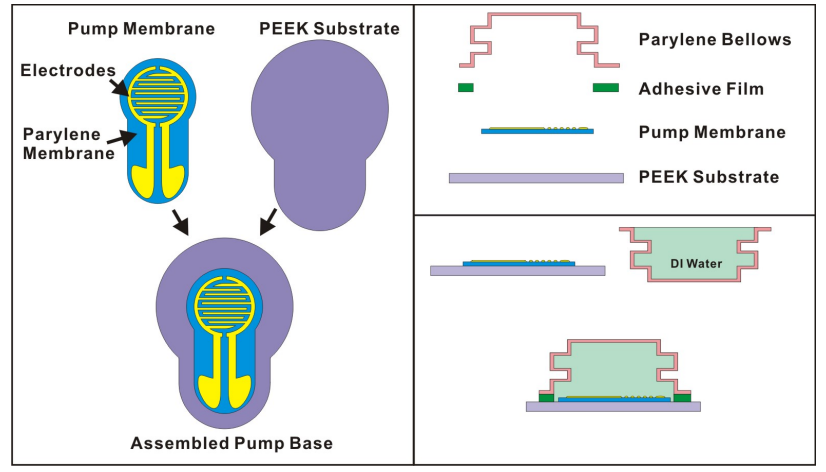

Figure 3: Parylene bellows actuator assembly process: (left) top view of electrode base assembly, (right) cross section of actuator components (top) and the assembly process (bottom).

\section{Integrated Valve}

Dual regulation passive check valves that permit forward flow only over a predetermined pressure range were integrated into the cannula [6]. The valve consists of four stacked disks (valve seat, pressure responsive valve plate, spacer plate, and pressure limiter) packaged in fluorinated ethylene propylene (FEP) heat-shrink cannula without the use of any adhesives.

\section{Passive Pump Assembly}

Passive pumps were assembled (Figure 4). These units did not contain the electrochemical bellows actuator or valve and consisted of the silicone rubber/PEEK base, cannula, and silicone reservoir cavity. Without the actuator, these implanted pumps provided drug by diffusion from the reservoir through the cannula.

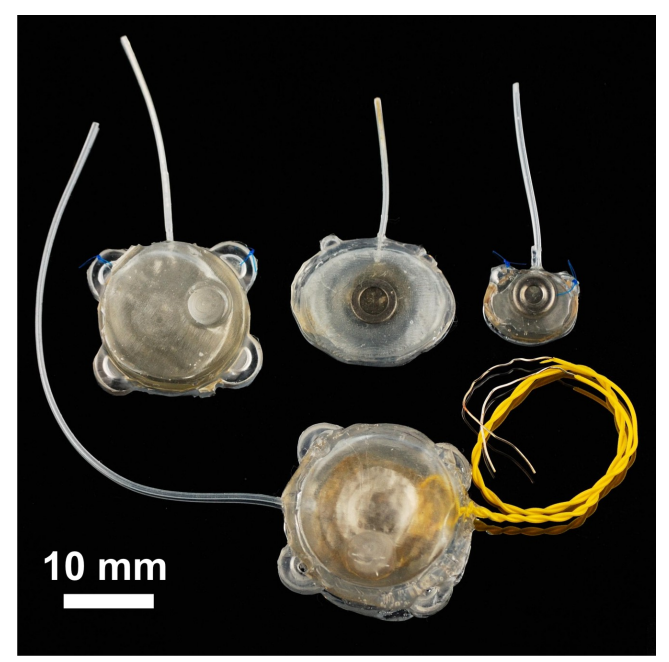

Figure 4: Photographs of active and passive drug delivery systems: (bottom) active device and (top row) passive devices.

\section{Active Pump Assembly}

All parts required to assemble the cancer drug delivery pump are shown in Figure 5. The assembled bellows actuator was mounted onto a silicone rubber (MDX44210, Dow Corning) and PEEK base with an integrated cannula (silicone rubber, $0.305 \mathrm{~mm}$ ID x $0.610 \mathrm{~mm}$ OD, VWR International) and then fully encapsulated by an overlying silicone rubber reservoir cavity. A small protruding bump on the reservoir served as a refill port and allows port location by palpation through the skin. Furthermore, the refill port was offset from the bellows below to prevent puncture of the actuator bellows by the refill needle. All silicone rubber parts were fabricated by casting from laser-machined acrylic molds.

The pump used for the cancer studies did not include a valve. A smaller intraocular drug pump for glaucoma therapy integrated a valve at the tip of the cannula to prevent accidental dosing and backflow of fluids (Figure 6 ). The valve module was inserted into a short silicone rubber cannula attached to the pump housing to complete the assembly of the valved pump. 


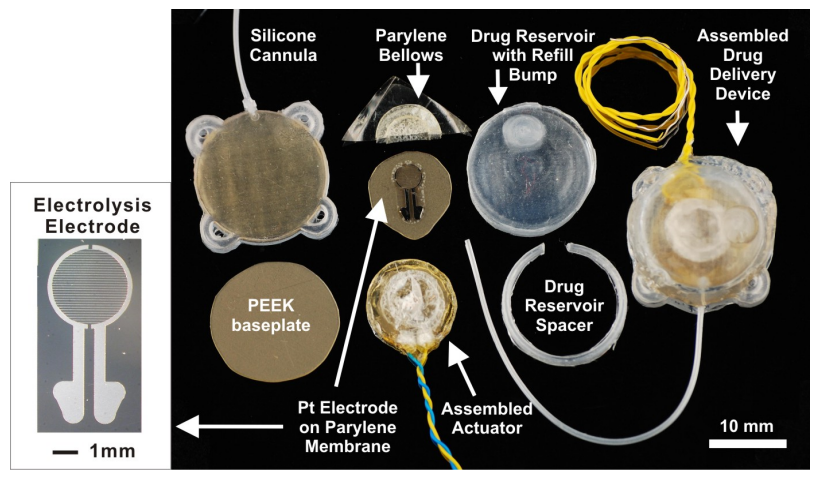

Figure 5: Drug delivery device components for assembly including assembled cancer drug delivery device for small animals (far right) and (left) a close-up of the electrolysis electrodes prior to release from silicon substrate.
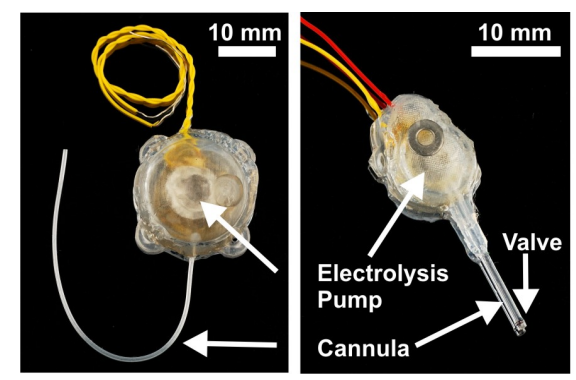

Figure 6: Photographs of fabricated drug delivery systems: (left) device used for cancer studies and (right) device to be used for intraocular drug delivery. The dark metal ring in the intraocular device is used to locate the refill port through the conjunctiva (part of the eye wall).

\section{EXPERIMENTS AND RESULTS}

\section{Electrolysis Electrodes}

Previously reported $\mathrm{Pt}$ electrodes deposited on glass substrates $[3,5]$ were robust up to currents greater than 5 mA (data not shown). Pt electrodes on Parylene, however, exhibit delamination even for modest applied currents. Improvement in $\mathrm{Pt}$ adhesion to Parylene was obtained following annealing $\left(150{ }^{\circ} \mathrm{C}\right.$ and $\left.205{ }^{\circ} \mathrm{C}\right)$ and a more aggressive descum prior to metal deposition. Electrodes having a $\mathrm{Ti}$ adhesion layer were also fabricated and a subset were annealed $\left(200{ }^{\circ} \mathrm{C}\right)$. Overall, the greatest improvement in electrode adhesion under applied current was attained by inclusion of the $\mathrm{Ti}$ adhesion layer but without subsequent annealing of the metal/Parylene composite film.

Electrodes were also characterized using electrochemical impedance spectroscopy (EIS) (Gamry Reference 600 Potentiostat and EIS300 Software, Gamry Instruments) performed in $1 \mathrm{X}$ phosphate buffered saline before and after application of current $(0.2,0.6$, and 1.0 $\mathrm{mA}$ were applied successively to the same $\mathrm{Pt} / \mathrm{Ti}$ electrodes). As expected, the impedance decreased with increasing frequency (Figure 7). After the first application of (low) current, the impedance decreased at lower frequencies and exhibited little change for increasing current. This behavior was consistent across the tests in electrodes and indicates a change in the effective surface area that is likely a result of electrolysis. This phenomenon is not fully understood and is the subject of further investigation.

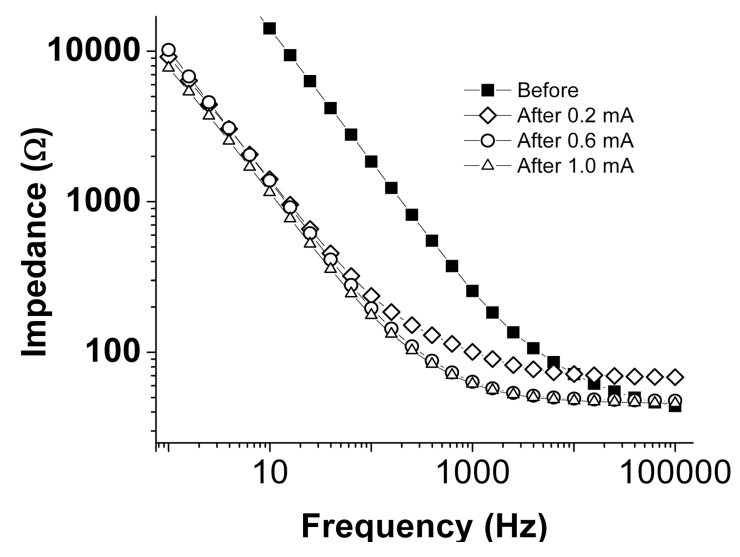

Figure 7: Electrochemical impedance spectroscopy results for the Pt-Ti electrode before and after current application.

\section{Fluid Dynamic Testing}

Stand-alone electrodes and fully packaged pumps were tested at the benchtop prior to implementation in animals. Temporal control of drug delivery was successfully demonstrated. Stand-alone electrodes (without bellows) were fixed in a custom laser-machined (Mini/Helix 8000, Epilog, Golden, CO) acrylic test fixture filled with deionized water. The application of constant current (2400 Sourcemeter, Keithley, Instruments Inc., Cleveland, $\mathrm{OH}$ ) induced electrolysis, which dispensed fluid through the fixture output and into a calibrated micropipette (AccuFill 90, Becton, Dickinson and Company, NJ). Flow rates for the applied currents were observed with a computercontrolled CCD camera (PL-A662, PixeLINK, Ottawa, Canada) attached to a microscope. Figure 8 displays the flow rates generated when current is applied to the $\mathrm{Pt} / \mathrm{Ti}$ electrodes. A similar method was use to obtain flow rates and delivered volume from the assembled pumps (inset Figure 8).

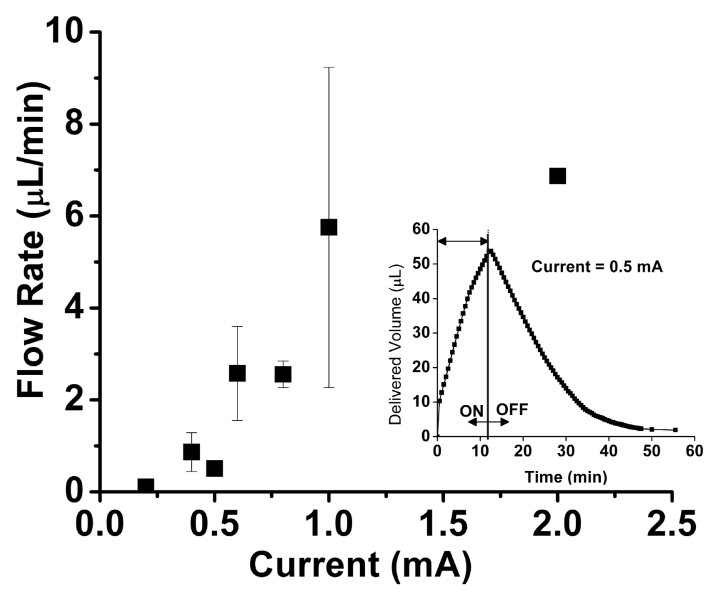

Figure 8: Electrolysis-induced flow rate $(\mu \mathrm{L} / \mathrm{min})$ in $\mathrm{Pt} / \mathrm{Ti}$ electrodes (mean $\pm \mathrm{SE}$ ). Inset shows delivered volume versus time for an assembled pump (0.5 mA).

\section{In Vivo Drug Delivery}

HT29 cells were implanted subcutaneously in both sides of the flank in nude mice to induce tumors (Figure 1). Implanted pumps (without a flow regulation valve) (Table 
1) were operated under constant current $(0.78 \mathrm{~mA})$ using a custom battery-powered pack (Figure 9). Reservoirs were placed subcutaneously up the middle of the back of the nude mice and the cannula tip was directed to the left tumor. The right side tumor was untreated.

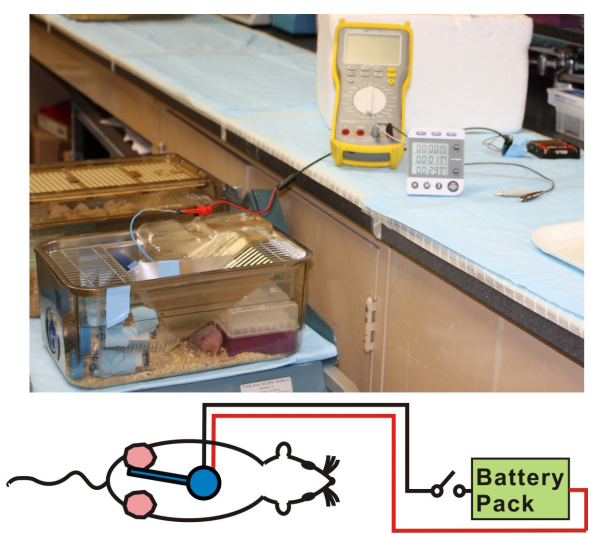

Figure 9: Photograph of in vivo testing setup and illustration showing battery pack connection to pumps.

Table 1. Cancer drug delivery device specifications.

\begin{tabular}{|l|l|}
\hline Components & Dimensions \\
\hline Drug Reservoir & Diameter $=18 \mathrm{~mm}$ \\
& Height $=3.5 \mathrm{~mm}$ \\
& Volume $=560 \mu \mathrm{L}$ \\
\hline Cannula & Length $=$ cut on site \\
& $\begin{array}{l}\text { ID }=0.305 \mu \mathrm{mm} \\
\text { OD }=0.610 \mathrm{~mm}\end{array}$ \\
\hline Pump Electrodes & Width $=20 \mu \mathrm{m}$ \\
& Gap $=100 \mu \mathrm{m}$ \\
\hline
\end{tabular}

Passive pumps (without the bellows actuator) were also implanted and continuously delivered phosphate buffered saline, gold nanorods, free siRNA, or nanoplexes via diffusion. Free siRNA and nanoplexes were also delivered by pumping daily for two weeks $(\sim 50 \mu \mathrm{L}$ bolus infusion). Pumps were refilled two times per week. A subset of treated tumors was exposed to radiation (100 $\mathrm{rad})$. Tumors were periodically monitored and then removed at the conclusion of the study. Active delivery of the nanoplexes in combination with radiation therapy showed significant tumor regression ( 50\%) over diffusion and injection based delivery (Figure 10), with the greatest effect observed adjacent to the delivery site.

At the conclusion of the in vivo studies, devices were explanted and re-tested. Evaluation under the CCD camera verified that two of the three devices still functioned (data not shown). The third device exhibited a perforation in the bellows and some delamination of the electrodes; the exact cause and time of failure are unknown but may be attributed to the explant procedure.

\section{CONCLUSION}

We successfully demonstrated integrated drug delivery systems consisting of an electrochemical bellows actuator, a transcutaneous cannula, and a dual regulation valve. Future work includes in vivo studies with improved, delamination-resistant electrodes and wireless actuation.
Additional animal studies are planned including more cancer radiation reduction experiments and intraocular delivery for the treatment of glaucoma to prevent blindness using a valved pump.

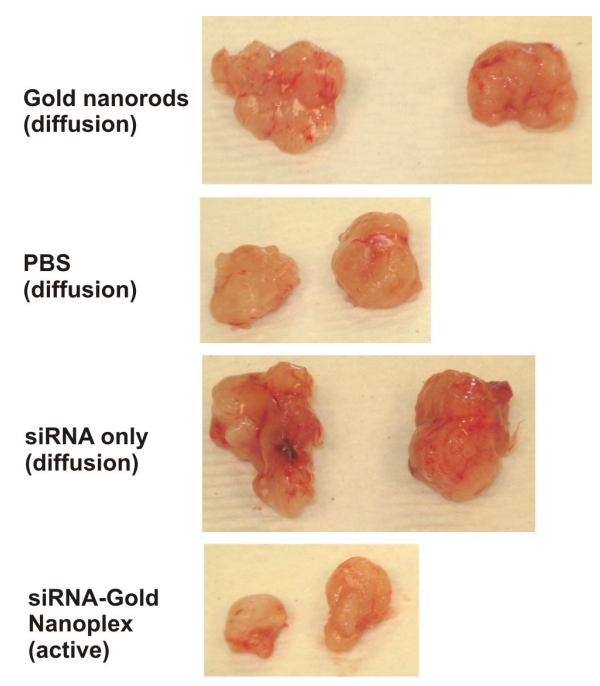

Figure 10: Photographs comparing size of the tumors after delivery of the controls and nanoplexes from a pump. The treated tumor is on the left.

\section{ACKNOWLEDGEMENTS}

This work was funded in part by the NIH/NEI under award number R21EY018490 and a Wallace H. Coulter Foundation Early Career Translational Research Award. The authors would like to thank Dr. Donghai Zhu, Mr. Jun Seob Shin, and the members of the USC Biomedical Microsystems Laboratory for their assistance with this project.

\section{REFERENCES}

[1] E. Bonhoure, D. Pchejetski, N. Aouali, H. Morjani, T. Levade, T. Kohama, and O. Cuvillier, "Overcoming MDR-associated chemoresistance in HL-60 acute myeloid leukemia cells by targeting shingosine kinase-1" Leukemia, 2005, pp. 1-8

[2] D.M. Dykxhoorn, D. Palliser, and J. Lieberman, "The silent treatment: siRNAs as small molecule drugs" Gene Therapy, vol. 13, 2006, pp. 541-552

[3] P.Y. Li, R. Sheybani, J.T.W. Kuo, and E. Meng, "A Parylene Bellows Electrochemical Actuator for Intraocular Drug Delivery" Proc. of Transducers, Denver, Colorado, USA, Jun. 21-25, 2009, pp. 1461-1464

[4] P.Y. Li, J. Shih, R. Lo, R. Agrawal, S. Saati, M.S. Humayun, Y.C. Tai, and E. Meng, "An Electrochemical Intraocular Drug Delivery Device" Sensors and Actuators A: Physical, vol. 143, Issue 1, pp. 41-48, 2008

[5] P.Y. Li, R. Sheybani, C. Gutierrez, J.T.W. Kuo, and E. Meng, "A Parylene Bellows Electrochemical Actuator" IEEE/ASME Journal of Microelectromechanical Systems, 2009 (accepted)

[6] R. Lo and E. Meng "In-Plane Bandpass Regulation Check Valve in Heat-Shrink Packaging for Drug Delivery" Proc. of IEEE Micro Electro Mechanical Systems, Sorrento, Italy, Jan. 25-29, 2009, pp. 236-239 